\title{
The pathways from distress tolerance to Cyberchondria: A multiple-group path model of young and middle adulthood samples
}

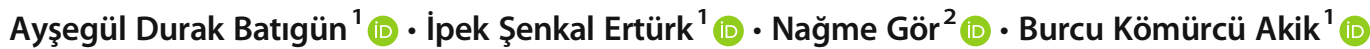

Published online: 5 September 2020

(C) Springer Science+Business Media, LLC, part of Springer Nature 2020

\begin{abstract}
The use of the Internet for medical information elicited a recent term called "cyberchondria". This study aimed to scrutinize the mediating effects of health anxiety (HA), anxiety symptoms (AS), and Internet addiction (IA) in the pathway from distress tolerance (DT) to cyberchondria by using a bootstrapping method. In order to examine the role of age in the proposed model, multiple-group path analysis was used to evaluate differences between young and middle adulthood groups. The final sample consisted of both young adult $(n=209)$ and middle adult $(n=221)$ Internet users located in Ankara, Turkey. The results of path analyses for both age groups showed that DT is negatively associated with AS and HA; AS and HA are positively associated with IA; IA and HA are positively associated with cyberchondria. Mediation analysis for both age groups demonstrated that AS and HA significantly mediated the relationship between DT and IA; IA significantly mediated the relationships of AS and HA with cyberchondria; HA significantly mediated the relationship between DT and cyberchondria. The results of the multiple-group path analysis showed that the relationship between IA and cyberchondria is significantly stronger in middle adulthood than young adulthood. The results of the current study are consistent with the relevant literature and provide crucial contribution especially by focusing on the role of age.
\end{abstract}

Keywords Cyberchondria $\cdot$ Health anxiety $\cdot$ Anxiety symptoms $\cdot$ Distress tolerance $\cdot$ Internet addiction

The Internet has been evaluated as the largest medical library in the World (Morahan-Martin 2004) as people reach Web sites that include the symptoms of diseases, information on risks and benefits of drugs, treatment methods or the opinions of professionals, and they can also have contact with other Internet users through chat rooms or social media (De Choudhury et al. 2014; Epstein 2017; Greene and Kesselheim 2010; Starcevic and Berle 2013). Hence, the Internet cannot be underestimated when researchers conduct studies on health.

The use of the Internet for health elicited a phenomenon called as "cyberchondria" which is characterized by an

Burcu Kömürcü Akik

komurcu@ ankara.edu.tr

1 Department of Psychology, Faculty of Languages History and Geography, Ankara University, PC: 06100 - Sihhiye, Ankara, Turkey

2 Department of Psychology, School of Humanities and Social Sciences, Istanbul Medipol University, Kavac1k South Campus, Göztepe Mahallesi Atatürk Caddesi No 40/16 PC: 34815 Beykoz, Istanbul, Turkey excessive and escalated seek for health-related information on the Web while feeling increased distress or anxiety, and a need to get reassurance (Starcevic 2017; Starcevic and Berle 2013; White and Horvitz 2009). For people suffering from cyberchondria, this repeated medical information search for themselves or for others would lead them to gain considerable amount of information that is hard to interpret on one's own, and then would result in reading about serious health outcomes with an increased anxiety (Aiken and Kirwan 2013; Lutwak 2017; Starcevic and Berle 2013).

Cyberchondria is closely related to hypochondria (Starcevic and Berle 2013) in which the misinterpretation of bodily symptoms leads to the belief that one has a severe illness (APA 2000). Health anxiety (HA) (i.e., tendency to misinterpret uncertain health-related information as a proof for a severe physical illness; Salkovskis et al. 2003) was evaluated as a distinct (Mathes et al. 2018) and pivotal feature of cyberchondria (Starcevic and Berle 2013). Previous studies has shown that as the severity of HA increases, individuals could conduct more online health-related research (te Poel et al. 2016), and they could feel worse or more anxious after symptom checking on the Web (Doherty-Torstrick et al. 2016; Muse et al. 2012; Singh and Brown 2016). 
As cyberchondria is linked to distress or anxiety related to health (Starcevic and Berle 2013), distress tolerance (DT) should be considered when studying cyberchondria. DT was defined as "the capacity to experience and withstand negative psychological states" (Simons and Gaher 2005, pp. 83). In the literature, the components of DT (i.e., intolerance of uncertainty, ambiguity, frustration, negative emotional states, and physical sensations) were found to be significantly correlated with HA (Fergus et al. 2015). Especially, intolerance of uncertainty was viewed as an outstanding risk factor for anxiety (Carleton 2012), and the positive correlation between intolerance to uncertainty and HA was found by Kraemer et al. (2016). Furthermore, anxiety sensitivity and intolerance of uncertainty were evaluated as the probable risk factors for cyberchondria (Fergus 2013; Fergus 2015; Norr et al. 2015; Zangoulechi et al. 2018).

Another precursor of cyberchondria could also be Internet addiction (IA) (e.g., Ivanova 2003). Internet addiction has been perceived as an impulse control disorder (Young 2009). In this regard, studies have shown that as the time spent for online health-related search gets longer, functional impairment and anxiety during/after the search increases (DohertyTorstrick et al. 2016); and individuals with higher IA also reported higher scores on HA and two aspects of cyberchondria (i.e., escalations and persistence of concern) (Ivanova 2013). In another study, the participants who experienced increased HA after online medical information search reported significantly greater problematic Internet use (PIU) (Fergus and Dolan 2014). Consistently, the positive correlation between cyberchondria and PIU was supported by Fergus and Spada (2017). It has also been reported that individuals with higher HA use the Internet for health-related searches for a longer time and more often (Baumgartner and Hartmann 2011; Muse et al. 2012).

Research regarding the role of demographic variables on online health information search is still relatively nascent. Considering age, in the study of German version of the Cyberchondria Severity Scale (CSS), the total score and age were not correlated, but "the mistrust of health professionals" subscale scores have increased as people get older (Barke et al. 2016). In parallel, no significant relationship was found between age and laypeople's evaluations on their activities and experiences while using the Internet for their symptoms (White and Horvitz 2009).

The current study proposes that a decrease in DT is associated with an increase in AS and HA, which in turn leads to an increase in IA, and IA is associated with an increase in cyberchondria in young and middle adulthood. Additionally, the current study suggests that a decrease in DT is associated with an increase in HA, which also in turn leads to an increase in cyberchondria in young and middle adulthood. Furthermore, by considering the changes in health-related problems as people age, this study will contribute to the related literature by comparing the young and middle adulthood samples in terms of proposed model and thus exploring which associations are significantly stronger in the development of cyberchondria across samples.

To sum, the aim of the current study is to investigate the hypothesized mediation model in which (a) anxiety symptoms (AS) and HA would mediate the relationship between DT and IA, (b) IA would mediate the relationship of AS and HA with cyberchondria, (c) HA would mediate the relationship between DT and cyberchondria in both samples, (d) suggested paths are different across the two samples.

\section{Method}

\section{Sample and Procedure}

The study sample consisted of participants in their young and middle adulthood $(N$ : 442, age: $18-65, M=36.46, S D=$ 13.75). Data was recruited through convenience sampling technique (Dörnyei 2007) from Ankara, Turkey. Participants of the young ( $n$ : 209 , age: $18-35, M=23.82, S D=4.29)$ and the middle ( $n$ : 221 , age: $36-65, M=48.41, S D=7.48$ ) adulthood sample could use the Internet and had no psychiatric diagnosis. Since 8 participants did not report their age, their data was not included in the analyses. After outliers were excluded, statistical analyses were run for 430 participants. The required permission was obtained from Ankara University Ethics Committee, and informed consent was obtained from all participants. Descriptive statistics were demonstrated in Table 1.

\section{Measures}

Demographic Information Form It is a form prepared by the authors to acquire information about the participants' sociodemographic characteristics (e.g., age, gender, education, any psychiatric diagnosis within the last six months).

Cyberchondria Scale (CS) CS is a 28-item self-report and 5point Likert-type scale developed in Turkish (Durak-Batigun et al. 2018) to assess health-related information seeking behavior from the Internet. The CS includes five subscales: "anxiety-increasing factors", "compulsion/hypochondria", "anxiety-reducing factors", "physician-patient interaction" and "non-functional internet use". Sample items are "I think myself as a hypochondriac / valetudinarian", "Internet has helped me to understand the terminology / explanations provided by the physician" and "The Internet was useful in the initial diagnosis of the disease". Cronbach alpha for subscales was $.88, .83, .80, .80, .84$, respectively and .93 for the total score. Higher scores indicate greater cyberchondria. 
Table 1 Descriptive statistics of young adulthood and middle adulthood samples

\begin{tabular}{lllllllll}
\hline & \multicolumn{3}{c}{ Young adulthood sample } & \multicolumn{5}{c}{ Middle adulthood sample } \\
\hline & $n$ & $(\%)$ & $M$ & $S D$ & $n$ & $(\%)$ & $M$ & $S D$ \\
Age & 209 & 47.7 & 23.82 & 4.29 & 221 & 50.5 & 48.41 & 7.48 \\
Sex & & & & & & & & \\
Male & 93 & 44.5 & & & 100 & 45.2 & & \\
Female & 116 & 55.5 & & & 121 & 54.8 & \\
Internet usage 1-3 h in a day & 91 & 43.5 & & & 162 & 73.3 & \\
Online health research average per month & 129 & 61.7 & & & 107 & 48.4 & \\
\hline
\end{tabular}

Internet Addiction Test (IAT) IAT is a 20-item with a 6-point Likert type scale developed (Young 1998) and adapted into Turkish (Bayraktar 2001) to assess IA. A sample item is "How often do you find that you stay online longer than you intended?". Cronbach alpha was .91. Higher scores of the scale indicate higher IA. The scale has sufficient psychometric properties.

Health Anxiety Inventory (HAI) HAI is an 18-item with a 3point Likert type scale developed (Salkovskis et al. 2002) and adapted into Turkish (Karapıçak et al. 2012) to evaluate HA independent from the current health status. A sample item is “a) I do not worry about my health, b) I occasionally worry about my health, c) I spend much of my time worrying about my health, d) I spend most of my time worrying about my health". Participants are asked to select the one which best describes their feelings, over the past six months. Cronbach alpha was .91. Aydemir et al. (2013) also performed a reliability and validity of the scale in individuals with somatoform disorder, panic disorder and major depressive disorder. In their study, Cronbach alpha was .92. HAI was found valid and reliable.

Distress Tolerance Scale (DTS) DTS is a 15 -item with a 5-point Likert-type self-report scale developed (Simons and Gaher 2005) and adapted into Turkish (Sargin et al. 2012). The scale consisted of three subscales called "tolerance", "regulation" and "self-efficacy" (Sargin et al. 2012). A sample item is "Feeling distressed or upset is unbearable to me". Cronbach alpha for the total score is .89 and for the subscales it ranged between .64 and .90 . Higher scores indicate higher ability in DT. DTS was a valid and reliable instrument.

Brief Symptom Inventory (BSI) BSI is a 53-item self-report scale with a 4-point Likert type scale developed (Derogatis 1992) and adapted into Turkish (Șahin and Durak 1994) with three different studies to evaluate a variety of psychological symptoms. These studies reported that the scale consisted of five subscales called "anxiety", "depression", "negative self", "somatization" and "hostility". Sample items are "Feelings of guilt", and "The idea that something is wrong with your mind". Cronbach alpha for these subscales ranged between
.75 and .87 . Higher scores indicate higher frequency of symptoms of the individual. In the current study, anxiety subscale was used.

\section{Analytic Strategy}

All data analyses were performed using IBM SPSS Statistics 21 and AMOS software. Independent samples t test was calculated to examine differences between young and middle adulthood groups in terms of all study variables (Field 2013). Pearson's correlation analysis was carried out to investigate relationships between the study variables. Path analysis was used to test the hypothesized mediation model for both young and middle adulthood samples. The mediation effects were analyzed for both groups, using a bootstrapping method (2000 resamples) with $95 \%$ bias-corrected confidence intervals (BC CI) (Shrout and Bolger 2002). It is considered that if zero is not included in the CI for the estimate of the indirect effect this effect is statistically significant at $p<0.05$ level (Shrout and Bolger 2002). Furthermore, a multiple-group path analysis was employed to test whether structural weights were invariant across distinct groups (Byrne 2016).

\section{Results}

\section{Independent Samples T-Test and Correlation Analysis}

The results of independent samples $t$-test, there is significant difference between young and middle adulthood sample in terms of AS $(t=3.86, p<.001)$, IA $(t=9.16, p<.001)$, and cyberchondria $(t=2.60, p<.05)$. Pearson's correlation coefficients for each sample are presented in Table 2.

\section{Path Analysis for Young Adulthood and Middle Adulthood Samples}

A single-group path model was tested for young and middle adulthood samples separately. The fit indices for the model of each sample and the baseline model in which the two path models were combined into one model before and after the error association are presented in Table 3. 
Table 2 Correlation coefficients and Independent Samples T-Test

\begin{tabular}{|c|c|c|c|c|c|c|c|c|c|c|}
\hline & \multicolumn{2}{|c|}{ Young adulthood sample $(n=209)$} & \multicolumn{2}{|c|}{ Middle adulthood sample $(n=221)$} & \multirow[b]{2}{*}{$t$} & \multirow[b]{2}{*}{ DT } & \multirow[b]{2}{*}{ AS } & \multirow[b]{2}{*}{ HA } & \multirow[b]{2}{*}{ IA } & \multirow[b]{2}{*}{$\mathrm{C}$} \\
\hline & $M$ & $S D$ & $M$ & $S D$ & & & & & & \\
\hline DT & 49.99 & 11.49 & 51.85 & 11.27 & -1.69 & 1 & $-.36^{* *}$ & $-.36 * *$ & $-.23 * *$ & $-.24 * *$ \\
\hline AS & 9.81 & 7.72 & 7.09 & 6.89 & $3.86^{* *}$ & $-.23 * *$ & 1 & $.42 * *$ & $.43 * *$ & $.40 * *$ \\
\hline HA & 14.78 & 6.12 & 14.47 & 7.42 & .47 & $-.35 * *$ & $.24 * *$ & 1 & $.40 * *$ & $.50 * *$ \\
\hline IA & 46.11 & 15.86 & 34.04 & 10.83 & $9.16^{* *}$ & $-.23 * *$ & $.41 * *$ & $.37 * *$ & 1 & $.48 * *$ \\
\hline $\mathrm{C}$ & 56.53 & 14.71 & 52.60 & 16.63 & $2.60 *$ & $-.20 * *$ & $.34 * *$ & $.37 * *$ & $.39 * *$ & 1 \\
\hline
\end{tabular}

${ }^{*} p<.05, * * p<.001$. Correlations above the diagonal are for the group of middle adulthood; Those below the diagonal are for the group of young adulthood. DT: Distress tolerance, AS: Anxiety symptoms, HA: Health anxiety, IA: Internet addiction, C: Cyberchondria

Baseline model and the models of both samples had acceptable goodness of fit values after the error association (see Fig. 1 and Fig. 2).

Firstly, for the young adulthood sample, DT is significantly associated with AS $(\beta=-.23, p<.001)$ and HA $(\beta=-.35$, $p<.001)$. AS $(\beta=.34, p<.001)$ and HA $(\beta=.28, p<.001)$ are significantly associated with IA. IA $(\beta=.29, p<.001)$ and HA $(\beta=.27, p<.001)$ are significantly associated with cyberchondria.

Secondly, in the middle adulthood sample, DT is significantly associated with AS $(\beta=-.36, p<.001)$ and HA $(\beta=$ $-.36, p<.001)$. AS $(\beta=.32, p<.001)$ and $\operatorname{HA}(\beta=.26$, $p<.001)$ are significantly associated with IA. IA $(\beta=.33$, $p<.001)$ and HA $(\beta=.37, p<.001)$ are significantly associated with cyberchondria.

\section{Mediation Analyses}

To test the mediating effects in the pathway from DT to cyberchondria, $95 \%$ bias-corrected confidence intervals (BC $\mathrm{CI})$ were calculated for both groups, using a bootstrapping method with 2000 re-samples (Shrout and Bolger 2002). Estimates, standard errors, and CI's of mediation models are presented in Table 4.
In the young adulthood sample, AS $(\mathrm{B}=-.11, \mathrm{CI}:-.16$, $-.05, p<.01)$ and HA (B = -.14, CI: $-.23,-.07, p<.01)$ significantly mediated the relationship between DT and IA. Additionally, IA significantly mediated the relationships of AS (B $=.19$, CI: $.07, .32, p<.01)$ and HA (B $=.20$, CI: .08, $.33, p<.01)$ with cyberchondria. Furthermore, HA significantly mediated the relationship between DT and cyberchondria (B $=-.12, \mathrm{CI}:-.20,-.06, p<.01)$. For the middle adulthood sample, AS (B $=-.11, \mathrm{CI}:-.17,-.06, p<.01)$ and HA (B $=-.09$, CI: $-.15,-.04, p<.01)$ significantly mediated the relationship between DT and IA. Additionally, IA significantly mediated the relationships of $\mathrm{AS}(\mathrm{B}=.26, \mathrm{CI}$ : $.14, .40, p<.01)$ and HA (B $=.19, \mathrm{CI} . .09, .31, p<.01)$ with cyberchondria. Furthermore, HA significantly mediated the relationship between DT and cyberchondria $(\mathrm{B}=-.20, \mathrm{CI}$ : $-.29,-.11, p<.01)$.

\section{Multiple-Group Path Analysis}

Multiple-group path analysis was used to test whether there were differences in the final model between young and middle adulthood groups. The significance of the differences in the structural weights was investigated across the two samples. The unconstrained model (no constraints

Table 3 Model fitness index values for the samples of young adulthood and middle adulthood

\begin{tabular}{|c|c|c|c|c|c|c|c|c|c|}
\hline & & $\chi^{2}$ & $d f$ & $\begin{array}{l}\chi^{2} / \\
d f\end{array}$ & CFI & GFI & AGFI & RMSEA & ECVI \\
\hline \multirow[t]{3}{*}{ Model 1} & Total model (Baseline model) & 47.19 & 8 & 5.90 & .90 & .96 & .85 & .11 & $.21<1.00$ \\
\hline & Group 1 & 15.91 & 4 & 3.98 & .92 & .97 & .89 & .12 & $.18<.82$ \\
\hline & Group 2 & 31.28 & 4 & 7.82 & .89 & .95 & .81 & .18 & $.24<1.17$ \\
\hline \multirow[t]{3}{*}{ One error associated final model } & Total model (Baseline model) & 14.19 & 6 & 2.37 & .98 & .99 & .94 & .06 & $.15<1.00$ \\
\hline & Group 1 & 9.27 & 3 & 3.09 & .96 & .98 & .91 & .10 & $.16<.82$ \\
\hline & Group 2 & 4.92 & 3 & 1.64 & .99 & .99 & .96 & .05 & $.13<1.17$ \\
\hline
\end{tabular}

Group 1: young adulthood sample, Group 2: middle adulthood sample; CFI: comparative fit index, GFI: goodness-of-fit index, AGFI: adjusted goodness-of-fit index, RMSEA: root mean square error of approximation, ECVI: expected cross validation index 


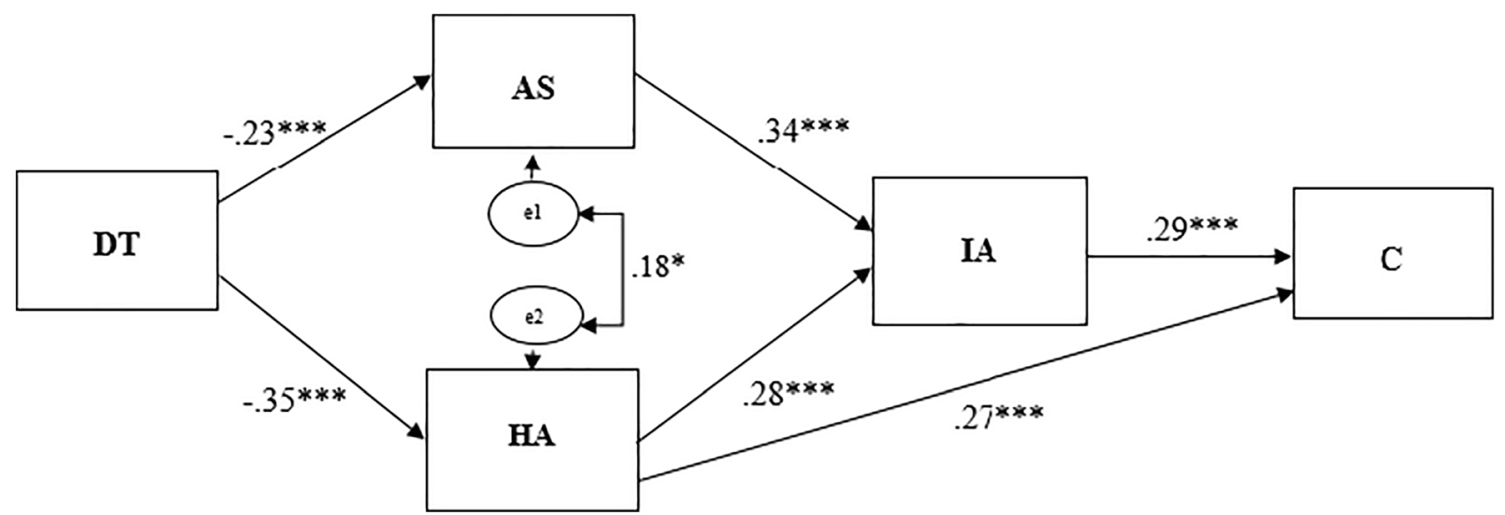

Fig. 1 Path Model for the group of young adulthood $(n=209) . * p<.05, * * * p<.001$. Standardized path coefficients among variables are presented. All path coefficients are statistically significant

across the groups) and the constrained model (the parameters are constrained equal across groups) were compared to test whether path coefficients are equal or invariant between two samples (Byrne 2016). Thus, it was tested whether there would be a significant difference between young and middle adulthood samples on each significant association in the path model. The Chi-square difference test was used to compare models (see Table 5).

Results showed that only the parameters coefficient in the path between IA and cyberchondria had a statistically significant difference between two samples $[\Delta \times 2(1)=4.693$, $p<.05]$. For the middle adulthood group, the standardized regression weight was $\beta=.330, p<.001$, whereas for the young adulthood group, it was $\beta=.289, p<.001$. This suggests that the relationship between IA and cyberchondria seems to be significantly stronger in middle adulthood than young adulthood. The other path coefficients were found to be invariant across the samples.

\section{Discussion}

The aim of the current study was (a) to investigate the hypothesized mediation model in which AS and HA would mediate the relationship between DT and IA in both young and middle adulthood samples (b) to investigate the hypothesized mediation model in which IA would mediate the relationship of AS and HA with cyberchondria in both samples (c) to investigate the hypothesized mediation model in which HA would mediate the relationship between DT and cyberchondria in both samples (d) to test whether study variables were different across the two samples.

Firstly, the results of path analyses for both age groups indicated that DT is negatively associated with AS and HA. DT was defined as the capacity to withstand repulsive situations such as perceived or actual negative affect or physical discomfort (Leyro et al. 2010). Since AS and HA could be considered as unpleasant feelings, our findings are coherent with the literature. In addition, our findings are consistent with the previous studies suggesting that DT is associated with several AS (Keough et al. 2010) and HA (Fergus et al. 2015). The second association was that AS and HA are positively associated with IA. Consistently, previous studies showed that anxiety positively predicted IA in an adolescent sample (Li et al. 2019) and anxiety was predicted by IA (Akin and Iskender 2011; Tang et al. 2014). It was also found that HA was correlated with some aspects of health-related Internet use, frequency and proportion of health-related online

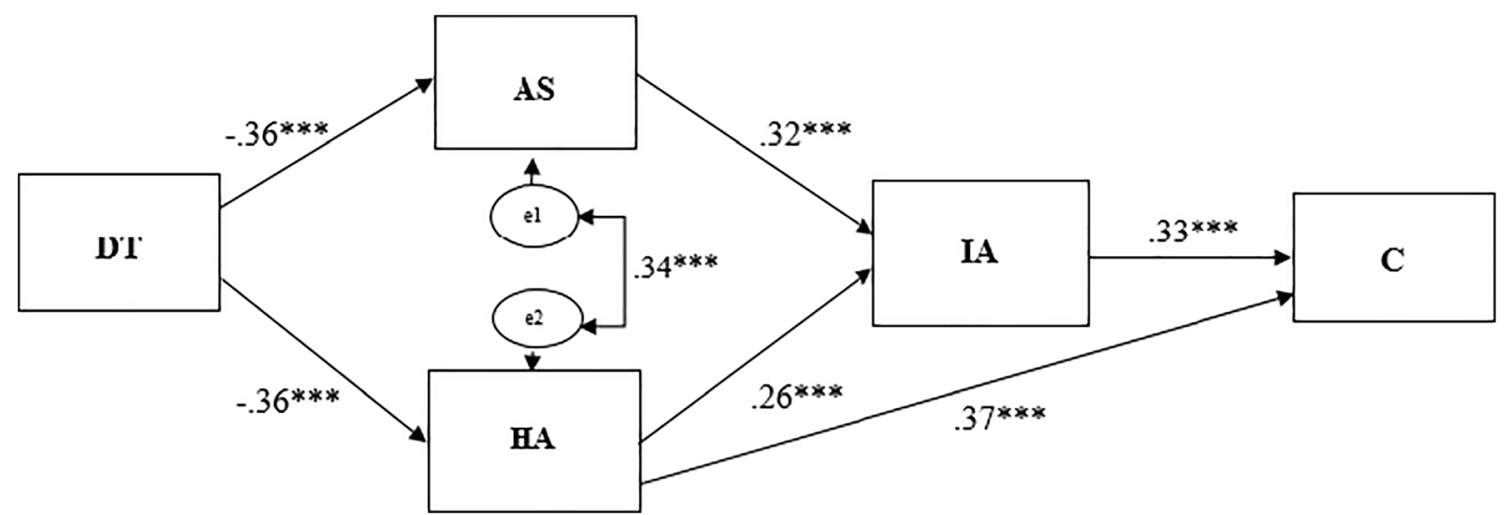

Fig. 2 Path Model for the group of middle adulthood $(n=221)$. *** $p<.001$. Standardized path coefficients among variables are presented. All path coefficients are statistically significant 
Table 4 Estimates of indirect effects

\begin{tabular}{|c|c|c|c|c|c|c|}
\hline & \multicolumn{3}{|c|}{ Young adulthood sample $(n=209)$} & \multicolumn{3}{|c|}{ Middle adulthood sample $(n=221)$} \\
\hline & $B(S E)$ & Lower & Upper & $B(S E)$ & Lower & Upper \\
\hline $\mathrm{DT} \rightarrow \mathrm{AS} \rightarrow \mathrm{IA}$ & $-.11 * *(.04)$ & -.16 & -.05 & $-.11 * *(.03)$ & -.17 & -.06 \\
\hline $\mathrm{DT} \rightarrow \mathrm{HA} \rightarrow \mathrm{IA}$ & $-.14 * *(.05)$ & -.23 & -.07 & $-.09 * *(.03)$ & -.15 & -.04 \\
\hline $\mathrm{AS} \rightarrow \mathrm{IA} \rightarrow \mathrm{C}$ & $.19 * *(.07)$ & .07 & .32 & $.26 * *(.08)$ & .14 & .40 \\
\hline $\mathrm{HA} \rightarrow \mathrm{IA} \rightarrow \mathrm{C}$ & $.20 * *(.08)$ & .08 & .33 & $.19 * *(.07)$ & .09 & .31 \\
\hline $\mathrm{DT} \rightarrow \mathrm{HA} \rightarrow \mathrm{C}$ & $-.12 * *(.04)$ & -.20 & -.06 & $-.20 * *(.05)$ & -.29 & -.11 \\
\hline
\end{tabular}

**p<.01. DT: Distress tolerance, AS: Anxiety symptoms, IA: Internet addiction, HA: Health anxiety, C: Cyberchondria, SE: Standard error searching, and time spent online for health purposes (Singh and Brown 2014). In a systematic review and meta-analysis, age explained some of the variation in the relationship between HA and cyberchondria (McMullan et al. 2019). McMullan et al. (2019) noted that previous studies that conducted with older participants found a stronger association between HA and cyberchondria. The results of these studies are consistent with our findings demonstrating that IA and HA are positively associated with cyberchondria. Our result is also consistent with the findings of the aforementioned studies (Fergus and Dolan 2014; Fergus and Spada 2017; Ivanova 2013). Briefly, it was found that all variables in the model were significantly associated with each other in the expected direction. Although there is no inference about the causality of the relationship, it can be said that there is a bidirectional relationship between using the Internet to seek health-related information and HA. Additionally, the interaction of IA with an anxious pattern seems to be a predisposition for cyberchondria in both age groups of our study.

Secondly, according to the mediation analyses, the results indicated that AS and HA mediated the relationship between DT and IA for both samples. It can be stated that low level of DT will increase the IA when AS and HA have mediator role in this relationship. A person with low level of DT may not

Table 5 Results of multiple-group path model

\begin{tabular}{lllll}
\hline & $\chi^{2}$ & $d f$ & $\Delta \chi^{2}$ & $\Delta d f$ \\
\hline $\begin{array}{l}\text { Total model (baseline model) } \\
\text { Models with following constraints }\end{array}$ & 14.191 & 6 & - & - \\
DT $\rightarrow$ AS & 15.450 & 7 & 1.259 & 1 \\
DT $\rightarrow$ HA & 15.029 & 7 & .838 & 1 \\
AS $\rightarrow$ IA & 15.603 & 7 & 1.411 & 1 \\
$\mathrm{HA} \rightarrow$ IA & 17.667 & 7 & 3.476 & 1 \\
$\mathrm{HA} \rightarrow$ C & 15.086 & 7 & .894 & 1 \\
$\mathrm{IA} \rightarrow$ C & 18.884 & 7 & $4.693^{*}$ & 1 \\
\hline
\end{tabular}

$*_{p}<.05$. DT: Distress tolerance, AS: Anxiety symptoms, IA: Internet addiction, HA: Health anxiety, C: Cyberchondria experience IA, however, one becomes predisposed to IA when accompanied by high scores of AS or HA. In addition, it can be inferred that individuals with higher AS and HA will have increased cyberchondria when IA has a mediator role. Taken together, these results support previous research findings showing that individuals with symptoms of AS or HA use the Internet to search for health-related information which may result in cyberchondria (e.g., Mathes et al. 2018; McMullan et al. 2019; Muse et al. 2012; te Poel et al. 2016; White and Horvitz 2009). In addition, our results are consistent with the findings of the previous studies conducted on IA/ PIU and cyberchondria (Fergus and Dolan 2014; Fergus and Spada 2017; Ivanova 2013). Furthermore, HA mediated the relationship between DT and cyberchondria. This result may indicate that people with low DT could develop cyberchondria when they have also high HA. Similarly, having difficulty in enduring uncertainty would result in increased HA and then health-related search on the Web (Fergus 2013; Zangoulechi et al. 2018). As our result shows, searching for certainty on the Internet could eventually lead to intense HA (Fergus 2013; Fergus and Spada 2017).

Thirdly, the results of multiple-group path analysis indicated that all direct associations between DT, AS, HA, IA (except for the association between IA and cyberchondria), and cyberchondria are invariant across young and middle adulthood samples. Only the association between IA and cyberchondria is stronger in the middle adulthood sample than young adulthood sample. This finding could suggest that individuals in middle adulthood use the Internet for different purposes than young adults, resulting in cyberchondria. In our study, since most of the middle adulthood sample use the Internet $1-3 \mathrm{~h}$ in a day ( $73.3 \%$ of the middle adulthood and $43.5 \%$ of the young adulthood), we only compared people who use the Internet $1-3 \mathrm{~h}$ in a day. In addition, $48.4 \%$ of the middle adulthood and $61.7 \%$ of the young adulthood sample searched online health-related information an average of 1-3 times per month. Hence, it was calculated that $35.5 \%$ of the middle adulthood sample and $26.8 \%$ of the young adulthood sample were searching online health-related information per month by using the Internet $1-3 \mathrm{~h}$ in a day. It may be 
interpreted that most of the participants in their middle adulthood spare more time to health-related search on Web during their relatively limited Internet usage time period. The fact that the middle adulthood group is more likely to face healthrelated problems (e.g., Hooker and Kaus 1994) may also contribute to this result. Considering their general health, they may be at risk in terms of cyberchondria. Although the Internet usage/addiction rates are high in young people (e.g., Cao and Su 2006; Lam et al. 2009), in our study the association between IA and cyberchondria is stronger in the middle adulthood sample compared to young adulthood sample. It should not be concluded that IA is higher in the middle adulthood group. In terms of age, our findings provide additional evidence for the role of IA in the onset and persistence of cyberchondria. The studies examining the relationships between cyberchondria and demographic variables (especially age) are quite limited and have conflicting results (Fox 2006; White and Horvitz 2009). Therefore, our findings provide profound contribution to the literature about the role of age in cyberchondria.

One of the strengths of our study is that it is the first study to test whether young and middle adulthood samples differ in the associations between IA and cyberchondria. Thus, considering age could be useful in intervention studies. Furthermore, given its association with distress and potential economic costs (Fergus 2014), identifying variables related to cyberchondria could be crucial for developing interventions and prevention strategies to address cyberchondria and factors that predispose to cyberchondria. According to Starcevic (2017), treatment approaches targeting cyberchondria should enable people to use the Internet for healthrelated purposes without increasing health concerns and reducing the time spent on health-related information online. When we investigated anxiety as a precursor of cyberchondria, consistent with the results of our study, it is considered important that individuals' levels of AS, HA, DT and IA should be also taken into account when developing cyberchondria interventions. In other words, our results spoke to the importance of taking into account psychological symptom levels, and age in any intervention efforts to reduce cyberchondria. In addition, other potential mediator or moderator variables that may influence the relationship between study variables and cyberchondria should be considered in interventions. Especially during COVID-19 pandemic, it is suggested that HA and cyberchondria serve as risk factors for coronavirus anxiety (Asmundson and Taylor 2020; Jungmann and Witthöft 2020). Our findings that point to roles of AS, HA, IA and DT in cyberchondria have significant importance for the context of pandemics.

Nevertheless, there are several limitations of our study. Firstly, more research is required to provide additional evidence whether the relationship between IA and cyberchondria is variant in different age groups. At this point, it is important to replicate the results with different samples to confirm these findings. Secondly, longitudinal study designs are needed to better understanding of the causal relationships. We defined structural paths among variables, but our study still does not provide any cause-effect relationships. As third, although the frequency of the Internet usage to obtain healthrelated information has been inquired in our study, a comprehensive investigation of the Internet usage purposes could help to have a deeper understanding about the differentiated paths in terms of age groups. Finally, since the study is based on self-reports only, structured interviews or behavioral measures could be helpful to understand cyberchondria from different perspectives.

Authors' Contributions All authors contributed to the study conception and design. Material preparation, data collection and analysis were performed by İpek Șenkal Ertürk, Nağme Gör, and Burcu Kömürcü Akik. The first draft of the manuscript was written by Ayșegül Durak Batıgün, İpek Șenkal Ertürk, Nağme Gör, and Burcu Kömürcü Akik and all authors commented on previous versions of the manuscript. All authors read and approved the final manuscript. All authors contributed equally to editing of the final manuscript.

Funding This study was supported by Ankara University Scientific Research Projects (Project No: 16B0649001).

Data availability The datasets analysed during the current study are available from the corresponding author on reasonable request.

\section{Compliance with Ethical Standards}

Conflicts of Interest/Competing Interests The authors declare that they have no conflict of interest.

Ethics Approval This study was performed in line with the principles of the Declaration of Helsinki. Approval was granted by the Ethics Committee of Ankara University.

Consent to Participate Informed consent was obtained from all individual participants included in the study.

Consent for Publication Not applicable.

Disclosure of Potential Conflicts of Interest On behalf of all authors, the corresponding author states that there is no conflict of interest.

Research Involving Human Participants and/or Animals All procedures performed in studies involving human participants were in accordance with the ethical standards of the institutional and/or national research committee (Ankara University Ethics Committee) and with the 1964 Helsinki declaration and its later amendments or comparable ethical standards.

Informed Consent Informed consent was obtained from all individual participants included in the study.

Code Availability Not applicable. 


\section{References}

Aiken, M., \& Kirwan, G. (2013). The psychology of cyberchondria and 'cyberchondria by proxy'. In A. Power \& G. Kirwan (Eds.), Cyberpsychology and new media: A thematic reader (pp. 158169). London and New York: Psychology Press Taylor \& Francis Group.

Akin, A., \& Iskender, M. (2011). Internet addiction and depression, anxiety and stress. International Online Journal of Educational Sciences, 3(1), 138-148.

American Psychiatric Association. (2000). Diagnostic criteria from $D S M-I V-T R$. Washington, DC: American Psychiatric Publishing.

Asmundson, G. J., \& Taylor, S. (2020). How health anxiety influences responses to viral outbreaks like COVID-19: What all decisionmakers, health authorities, and health care professionals need to know. Journal of Anxiety Disorders, 71, 102211.

Aydemir, Ö., Kırpınar, İ., Satı, T., Uykur, B., \& Cengisiz, C. (2013). Reliability and validity of the Turkish version of the health anxiety inventory. Archives of Neuropsychiatry, 50(4), 325-331.

Barke, A., Bleichhardt, G., Rief, W., \& Doering, B. K. (2016). The Cyberchondria severity scale (CSS): German validation and development of a short form. International Journal of Behavioral Medicine, 23, 595-605.

Baumgartner, S. E., \& Hartmann, T. (2011). The role of health anxiety in online health information search. Cyberpsychology, Behavior and Social Networking, 14(10), 613-618.

Bayraktar, F. (2001). The role of Internet usage in the development of adolescents [unpublished master's thesis]. Ege University, Izmir.

Byrne, B. M. (2016). Structural equation modeling with AMOS. Basic concepts, applications, and programming (Third ed.). New York: Routledge.

Cao, F., \& Su, L. (2006). Internet addiction among Chinese adolescents: Prevalence and psychological features. Child: Care, Health and Development, 33(3), 275-281.

Carleton, R. N. (2012). The intolerance of uncertainty construct in the context of anxiety disorders: Theoretical and practical perspectives. Expert Review of Neurotherapeutics, 12(8), 937-947.

De Choudhury, M., Morris, M. R., \& White, R. W. (2014). Seeking and sharing health information online: Comparing search engines and social media. In Proceedings of the 32nd annual ACM conference on human factors in computing systems (CHI'14) (pp. 1365-1376), New York, NY, USA: ACM.

Derogatis, L. R. (1992). BSI: Administration, scoring, and procedures manual-II. Towson, MD: Clinical Psychometric Research.

Doherty-Torstrick, E. R., Walton, K. E., \& Fallon, B. A. (2016). Cyberchondria: Parsing health anxiety from online behavior. Psychosomatics, 57(4), 390-400.

Dörnyei, Z. (2007). Research methods in applied linguistics: Quantitative, qualitative, and mixed methodologies. Oxford: Oxford University Press.

Durak-Batigun, A., Gor, N., Komurcu, B., \& Senkal-Erturk, I. (2018). Cyberchondria scale (CS): Development, validity and reliability study. Dusunen Adam The Journal of Psychiatry and Neurological Sciences, 31(2), 148-162.

Epstein, H. A. B. (2017). Cyberchondriacs. Journal of Hospital Librarianship, 17(4), 317-322.

Fergus, T. A. (2013). Cyberchondria and intolerance of uncertainty: Examining when individuals experience health anxiety in response to internet searches for medical information. Cyberpsychology, Behavior and Social Networking, 16(10), 735-739.

Fergus, T. A. (2014). The Cyberchondria severity scale (CSS): An examination of structure and relations with health anxiety in a community sample. Journal of Anxiety Disorders, 28, 504-510.

Fergus, T. A. (2015). Anxiety sensitivity and intolerance of uncertainty as potential risk factors for cyberchondria: A replication and extension examining dimensions of each construct. Journal of Affective Disorders, 184, 305-309.

Fergus, T. A., \& Dolan, S. L. (2014). Problematic internet use and internet searches for medical information: The role of health anxiety. Cyberpsychology, Behavior and Social Networking, 17(12), 761765.

Fergus, T. A., \& Spada, M. M. (2017). Cyberchondria: Examining relations with problematic internet use and metacognitive beliefs. Clinical Psychology \& Psychotherapy, 24(6), 1322-1330.

Fergus, T. A., Bardeen, J. R., \& Orcutt, H. K. (2015). Examining the specific facets of distress tolerance that are relevant to health anxiety. Journal of Cognitive Psychotherapy, 29(1), 32-44.

Field, A. (2013). Discovering statistics using IBM SPSS statistics (4th ed.). Los Angeles, London: SAGE.

Fox, S. (2006). Online health search 2006. Pew Internet \& American Life Project. https://www.pewinternet.org/2006/10/29/online-healthsearch-2006. Accessed 02 August 2019.

Greene, J. A., \& Kesselheim, A. S. (2010). Pharmaceutical marketing and the new social media. New England Journal of Medicine, 363(22), 2087-2089.

Hooker, K., \& Kaus, C. R. (1994). Health-related possible selves in young and middle adulthood. Psychology and Aging, 9(1), 126133.

Ivanova, E. (2013). Internet addiction and cyberchondria-their relationship with well-being. The Journal of Education, Culture, and Society, 1, 57-70.

Jungmann, S. M., \& Witthöft, M. (2020). Health anxiety, cyberchondria, and coping in the current COVID-19 pandemic: Which factors are related to coronavirus anxiety? Journal of Anxiety Disorders, 73, 102239.

Karapıçak, Ö. K., Aktaş, K., \& Aslan, S. (2012). Panik Bozukluğunda Sağlık Kaygısı Envanteri (Haftalık Kısa Form) Türkçe geçerlilik ve güvenilirlik çalışması. Klinik Psikiyatri, 15, 41-48.

Keough, M. E., Riccardi, C. J., Timpano, K. R., Mitchell, M. A., \& Schmidt, N. B. (2010). Anxiety symptomatology: The association with distress tolerance and anxiety sensitivity. Behavior Therapy, 41(4), 567-574.

Kraemer, K. M., O’Bryan, E. M., \& McLeish, A. C. (2016). Intolerance of uncertainty as a mediator of the relationship between mindfulness and health anxiety. Mindfulness, 7, 859-865.

Lam, L. T., Peng, Z. W., Mai, J. C., \& Jing, J. (2009). Factors associated with internet addiction among adolescents. Cyberpsychology \& Behavior, 12(5), 551-555.

Leyro, T. M., Zvolensky, M. J., \& Bernstein, A. (2010). Distress tolerance and psychopathological symptoms and disorders: A review of the empirical literature among adults. Psychological Bulletin, 136(4), 576-600.

Li, G., Hou, G., Yang, D., Jian, H., \& Wang, W. (2019). Relationship between anxiety, depression, sex, obesity, and internet addiction in Chinese adolescents: A short-term longitudinal study. Addictive Behaviors, 90, 421-427.

Lutwak, N. (2017). Commentary: Thoughts on cyberchondria-a new challenge for healthcare providers. Internet Journal of Allied Health Sciences and Practice, 15(3), 1-2.

Mathes, B. M., Norr, A. M., Allan, N. P., Albanese, B. J., \& Schmidt, N. B. (2018). Cyberchondria: Overlap with health anxiety and unique relations with impairment, quality of life, and service utilization. Psychiatry Research, 261, 204-211.

McMullan, R. D., Berle, D., Arnáez, S., \& Starcevic, V. (2019). The relationships between health anxiety, online health information seeking, and cyberchondria: Systematic review and meta-analysis. Journal of Affective Disorders, 245, 270-278.

Morahan-Martin, J. M. (2004). How internet users find, evaluate, and use online health information: A cross-cultural review. Cyberpsychology \& Behavior, 7(5), 497-510. 
Muse, K., McManus, F., Leung, C., Meghreblian, B., \& Williams, J. M. G. (2012). Cyberchondriasis: Fact or fiction? A preliminary examination of the relationship between health anxiety and searching for health information on the internet. Journal of Anxiety Disorders, 26(1), 189-196.

Norr, A. M., Albanese, B. J., Oglesby, M. E., Allan, N. P., \& Schmidt, N. B. (2015). Anxiety sensitivity and intolerance of uncertainty as potential risk factors for cyberchondria. Journal of Affective Disorders, 174, 64-69.

Şahin, N. H., \& Durak, A. (1994). Kısa Semptom Envanteri: Türk gençleri için uyarlanması. Türk Psikoloji Dergisi, 9, 44-56.

Salkovskis, P. M., Rimes, K. A., Warwick, H. M. C., \& Clark, D. M. (2002). The health anxiety inventory: Development and validation of scales for the measurement of health anxiety and hypochondriasis. Psychological Medicine, 32(5), 843-853.

Salkovskis, P. M., Warwick, H. M., \& Deale, A. C. (2003). Cognitivebehavioral treatment for severe and persistent health anxiety (hypochondriasis). Brief Treatment \& Crisis Intervention, 3(3), 353-367.

Sargın, A. E., Özdel, K., Utku, Ç., Kuru, E., Yalçınkaya Alkar, Ö., \& Türkçapar, M. H. (2012). Sıkıntıya Dayanma Ölçeği: Geçerlik ve güvenilirlik çalışması. Bilişsel Davranışçı Psikoterapi ve Arașttrmalar Dergisi, 1, 152-161.

Shrout, P. E., \& Bolger, N. (2002). Mediation in experimental and nonexperimental studies: New procedures and recommendations. Psychological Methods, 7(4), 422-445.

Simons, J. S., \& Gaher, R. M. (2005). The distress tolerance scale: Development and validation of a self-report measure. Motivation and Emotion, 29(2), 83-102.

Singh, K., \& Brown, R. J. (2014). Health-related internet habits and health anxiety in university students. Anxiety, Stress, \& Coping, 27(5), 542-554.
Singh, K., \& Brown, R. J. (2016). From headache to tumour: An examination of health anxiety, health-related internet use and 'query escalation'. Journal of Health Psychology, 21(9), 2008-2020.

Starcevic, V. (2017). Cyberchondria: Challenges of problematic online searches for health-related information. Psychotherapy and Psychosomatics, 86(3), 129-133.

Starcevic, V., \& Berle, D. (2013). Cyberchondria: Towards a better understanding of excessive health-related internet use. Expert Review of Neurotherapeutics, 13(2), 205-213.

Tang, J., Yu, Y., Du, Y., Ma, Y., Zhang, D., \& Wang, J. (2014). Prevalence of internet addiction and its association with stressful life events and psychological symptoms among adolescent internet users. Addictive Behaviors, 39(3), 744-747.

te Poel, F., Baumgartner, S. E., Hartmann, T., \& Tanis, M. (2016). The curious case of cyberchondria: A longitudinal study on the reciprocal relationship between health anxiety and online health information seeking. Journal of Anxiety Disorders, 43, 32-40.

White, R. W., \& Horvitz, E. (2009). Cyberchondria: Studies of the escalation of medical concerns in web search. ACM Transactions on Information Systems (TOIS), 27(4), 1-37.

Young, K. S. (1998). Internet addiction: The emergence of a new clinical disorder. Cyberpsychology \& Behavior, 1(3), 237-244.

Young, K. (2009). Internet addiction: Diagnosis and treatment considerations. Journal of Contemporary Psychotherapy, 39, 241-246.

Zangoulechi, Z., Yousefi, Z., \& Keshavarz, N. (2018). The role of anxiety sensitivity, intolerance of uncertainty, and obsessive-compulsive symptoms in the prediction of cyberchondria. Advances in Bioscience and Clinical Medicine, 6(4), 1-6.

Publisher's Note Springer Nature remains neutral with regard to jurisdictional claims in published maps and institutional affiliations. 